\title{
Morbidities of lung cancer surgery in obese patients
}

\author{
Binod Dhakal, MD, ${ }^{\text {a }}$ Daniel Eastwood, MS, ${ }^{\mathrm{b}}$ Sunitha Sukumaran, MD,${ }^{\mathrm{c}}$ George Hassler, MD ${ }^{\mathrm{d}}$ \\ William Tisol, MD, ${ }^{\mathrm{d}}$ Mario Gasparri, MD, ${ }^{\mathrm{d}}$ Nicholas Choong, $\mathrm{MD},{ }^{\mathrm{c}}$ and Rafael Santana-Davila, $\mathrm{MD}^{\mathrm{c}}$
}

Background: Obesity is a risk factor for increased perioperative morbidity and mortality in surgical patients. There have been limited studies to correlate the morbidity of lung cancer resection with obesity.

\begin{abstract}
Methods: We performed a retrospective study of patients who underwent surgical resection for lung cancer at the Medical College of Wisconsin, Milwaukee, from 2006 to 2010. Data on patient demographics, weight, pathological findings, and hospital course were abstracted after appropriate institutional review board approval. Perioperative morbidity was defined as atrial fibrillation, heart failure, respiratory failure, pulmonary embolism, or any medical complications arising within 30 days after surgery. The Fisher exact test was used to test the association between body mass index (BMI) and perioperative morbidities.
\end{abstract}

Results: Between 2006 and 2010, 320 lung resections were performed for lung cancer. The median age was 67 (interquartile range, 59-75) years, and 185 (57.8\%) were females. A total of 121 (37.8\%) of patients had a BMI lower than 25 , and $199(62.18 \%)$ patients had a BMI of 25 or higher. The 30 -day mortality rate was $1.8 \%(n=6)$ in the whole group; only 2 of these patients had a BMI of 25 or higher. Perioperative morbidity occurred in 28 $(23.14 \%)$ of patients with a normal BMI and in $47(23.61 \%)$ of patients with a BMI of 25 or higher $(P=.54)$. Specific morbidities encountered by patients with normal versus BMI of 25 or higher were as follows: atrial fibrillation, $11(9.09 \%)$ versus $24(12.06 \%)(P=.46)$; pulmonary embolism, $1(0.83 \%)$ versus $3(1.51 \%)$ $(P=1.0)$; congestive heart failure, $2(1.65 \%)$ versus $2(1.01 \%)(P=.63)$; renal failure, $4(3.3 \%)$ versus 2 $(1.0 \%)(P=.29)$; respiratory failure, $12(9.92 \%)$ versus $17(8.54 \%)(P=.69)$; and acute respiratory distress syndrome, $2(1.65 \%)$ versus $1(0.50 \%)(P=.55)$. The median hospital stay was 5 days in the lower BMI group and 4 days in the BMI of 25 or higher group $(P=.52)$.

Conclusions: Overweight and normal weight patients do not differ significantly in rates of perioperative morbidities, 30-day mortality, and length of stay. Our study indicates that potential curative surgical resections can be offered to even significantly overweight patients. (J Thorac Cardiovasc Surg 2013;146:379-84)

It is estimated that more than $65 \%$ of US residents are overweight or obese. ${ }^{1}$ Obesity is associated with many medical comorbidities, including hypertension, diabetes, and coronary artery disease. ${ }^{2,3}$ Its presence has been associated with an increased risk of cancers, including breast, colon, endometrium, and kidney. ${ }^{4,5}$ There is evidence that obese patients may also be at increased risk of developing lung cancer regardless of smoking status. ${ }^{5}$

Obesity is considered a risk factor for poor outcome in many surgical procedures, including urologic, gynecologic, cardiac, and pancreatic surgical procedures. ${ }^{6-8}$ However,

\footnotetext{
From the Divisions of General Internal Medicine ${ }^{\mathrm{a}}$ and Hematology and Oncology, ${ }^{\mathrm{c}}$ the Department of Cardio-Thoracic Surgery, ${ }^{\mathrm{d}}$ and the Division of Bio-Statistics, ${ }^{\mathrm{b}}$ Institute for Health and Society, Medical College of Wisconsin, Milwaukee, Wis. Supported, in part, by grant 1UL1RR031973 from the Clinical and Translational Science award program of the National Center for Research Resources, National Institutes of Health.

Disclosures: Authors have nothing to disclose with regard to commercial support.

Received for publication Aug 2, 2012; revisions received Jan 12, 2013; accepted for publication Feb 11, 2013; available ahead of print April 24, 2013.

Address for reprints: Rafael Santana-Davila, MD, Division of Hematology and Oncology, Medical College of Wisconsin, 9200 W Wisconsin Ave, Milwaukee, WI 53226 (E-mail: rsantana@mcw.edu).

0022-5223/\$0.00

Published by Elsevier Inc. on behalf of The American Association for Thoracic Surgery

http://dx.doi.org/10.1016/j.jtcvs.2013.02.083
}

this issue remains controversial, because it has not been consistent with all studies; some studies indicate that in noncardiac surgery, obesity alone is not a risk factor for perioperative morbidity and mortality. ${ }^{8-11}$

Not much is known about the effects of an elevated body mass index (BMI) on surgical morbidity and mortality after the resection of lung cancers. However, it is commonly and widely assumed that obese patients are at higher risk of surgical complications than those who are not obese. Many studies assessing the outcomes have included obesity as one variable with little comment on its prognostic significance. ${ }^{12,13}$ Whether higher BMI is associated with worse outcomes in patients undergoing lung cancer resections has been poorly studied. ${ }^{14-17}$ To our knowledge, there have been only 3 published studies on the subject. In a retrospective review of 499 patients who underwent resections for non-small cell lung cancer, a higher BMI was not associated with increased incidence of perioperative complications, mortality, or length of stay. ${ }^{18}$ In contrast, 2 retrospective studies have shown no effect on mortality but a higher incidence of intrathoracic complications. ${ }^{19,20}$

To further describe the effects of a higher BMI in patients undergoing resection of lung cancer, we performed a retrospective study to assess the impact of obesity on 


\section{Abbreviations and Acronyms \\ $\mathrm{BMI}=$ body mass index \\ $\mathrm{CI}=$ confidence interval \\ COPD $=$ chronic obstructive pulmonary disease \\ $\mathrm{FEV}_{1}=$ forced expiratory volume in 1 second \\ $\mathrm{OR}=$ odds ratio}

perioperative complications and mortality after resections for lung cancer.

\section{METHODS}

We performed a retrospective study of patients undergoing resection for lung cancer at The Medical College of Wisconsin, Milwaukee. After obtaining appropriate institutional review board approval, data from patients, who underwent lung cancer resection from January 2006 to December 2010, were abstracted. Resections of lung metastasis or benign tumors were excluded.

Patient demographics were collected, including age, sex, height, and weight. Patient variables and outcomes were stratified by BMI into 2 groups: lower than 25 and 25 or higher. Subjects were staged according to the 2010 American Joint Committee on Cancer staging. Outcomes measured included mortality within the 30 days of surgery, length of stay, and in-hospital complications. The in-hospital complications were defined as atrial fibrillation, heart failure, respiratory failure, pulmonary embolism, or any medical complications arising within 30 days of surgery. Respiratory failure was defined as any condition of respiratory distress from severe chronic obstructive pulmonary disease (COPD) or pneumonia that requires close monitoring, including noninvasive and invasive ventilation.

Patients were admitted on the day of surgery unless they required preoperative management of medical comorbidities. One of 3 boardcertified thoracic surgeons performed all the procedures. The prophylaxis for venous thromboembolism and the management of postoperative pain was performed as per standard guidelines. The duration of hospital stay was calculated from the day of surgery until the patient was discharged. The duration of chest tube was calculated from the day the chest tube was placed (on the day of surgery) until the tube was taken out, which may happen even after discharge in cases of an air leak. We defined mortality as any death occurring within 30 days after surgery.

\section{Statistical Analysis}

Association for BMI and hospital days was tested using the Wilcoxon rank sum test and Pearson and Spearman correlation. The Fisher exact test was used to test the dichotomous association between BMI of 25 or higher and the presence of perioperative morbidities. Odds ratios (ORs) are given with $95 \%$ exact confidence intervals (CIs). The log-rank test was used to compare the overall mortality between the groups.

Multivariate logistic regression of factors predicting perioperative morbidities was considered for all types of morbidity combined and for specific types in which there were more than 25 events. None of these multivariate models indicate more than a single predictive factor for any of the morbidities considered.

The analysis was performed using SAS, version 9.2 (The SAS Institute, Cary, NC).

\section{RESULTS}

\section{Patient Characteristics}

Between 2006 and 2010, there were 320 lung resections for non-small cell lung cancer. The patient baseline
TABLE 1. Baseline characteristics, surgery type, and pathology

\begin{tabular}{lccc}
\hline \multicolumn{1}{c}{ Variable } & BMI $<\mathbf{2 5}$ & BMI $>=\mathbf{2 5}$ & $\boldsymbol{P}$ value \\
\hline Characteristics & & & \\
$\quad$ Sex & & & .632 \\
$\quad$ Female & $72(59.50)$ & $113(56.78)$ & \\
$\quad$ Male & $49(40.50)$ & $86(43.22)$ & \\
Age, y & & & .96 \\
$\quad$ Median & 67 & 67 & \\
$\quad$ Range & $25-88$ & $44-85$ & \\
Surgery type & & & .61 \\
Lobectomy & $76(62.81)$ & $131(65.83)$ & \\
Wedge resection & $38(31.40)$ & $61(30.65)$ & \\
Pneumonectomy & $7(5.79)$ & $7(3.52)$ & \\
Median (range) tumor size, cm & $2.3(0.5-10)$ & $2.4(0.6-7)$ & .32 \\
Pathology & & & .0817 \\
Adenocarcinoma & $52(16.25)$ & $111(34.6)$ & \\
Squamous cell carcinoma & $50(15.62)$ & $57(17.8)$ & \\
Large-cell carcinoma & $6(1.8)$ & $13(4.06)$ & \\
$\quad$ Mixed type & $10(3.1)$ & $21(6.5)$ & \\
\hline
\end{tabular}

Values are given as number (percentage), unless otherwise indicated. $B M I$, Body mass index.

demographics are shown in Table 1. The median age was 67 (interquartile range, 59-75) years, and the sample consisted of $135(44.2 \%)$ men and $185(55.7 \%)$ women. The frequencies of surgical procedures were as follows: wedge resections, 99 patients $(32.7 \%)$; lobectomies, 207 patients $(62.9 \%)$; and pneumonectomies, 14 patients $(4.3 \%)$. Adenocarcinoma was the most prevalent histological feature present (138 patients, $43.9 \%$ ), followed by squamous cell carcinoma (107 patients, 31.6\%), bronchoalveolar carcinoma (25 patients, 7.1\%), large-cell carcinoma (19 patients, 5.4\%), and others (31 patients, $11.7 \%$ ). The median tumor size was $2.3 \mathrm{~cm}$ (interquartile range, 1.5 $3.9 \mathrm{~cm}$ ) and was highest in the pneumonectomy group. Of 14 pneumonectomies, 8 were left sided and the remaining 6 were right sided.

The distribution of patients in different BMI groups is shown in a histogram in Figure 1. Most patients were overweight $(124,38.7 \%)$, defined as a BMI between 25 and

\section{BMI distribution}

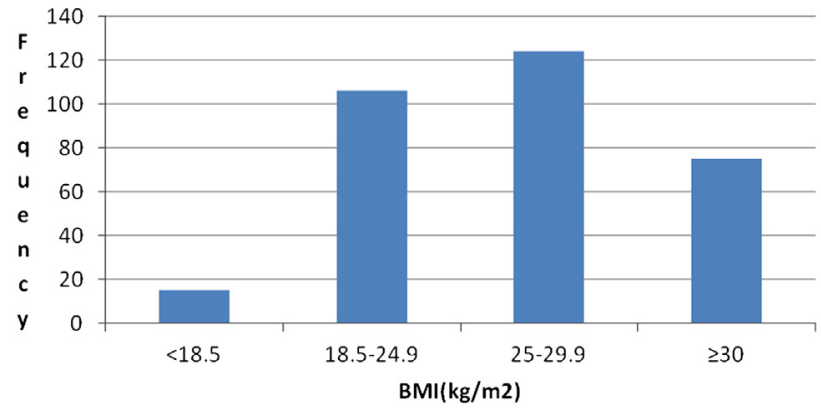

FIGURE 1. Body mass index (BMI) distribution as per World Health Organization classification. 
TABLE 2. Morbidities during the perioperative period

\begin{tabular}{lrlr}
\hline \multicolumn{1}{c}{ Morbidity } & BMI $<\mathbf{2 5}$ & BMI $\geq \mathbf{2 5}$ & $\boldsymbol{P}$ value \\
\hline All & $28(8.75)$ & $47(14.6)$ & .54 \\
Atrial fibrillation & $11(9.09)$ & $24(12.06)$ & .46 \\
Respiratory failure & $12(9.92)$ & $17(8.54)$ & .69 \\
Pulmonary embolism & $1(0.83)$ & $3(1.51)$ & 1.00 \\
CHF exacerbation & $2(1.65)$ & $2(1.01)$ & .63 \\
Acute respiratory distress & $2(1.65)$ & $1(0.50)$ & .55 \\
$\quad$ syndrome & & & \\
Renal failure & $4(3.3)$ & $2(1.0)$ & .29 \\
30-d Mortality & $4(1.25)$ & $2(0.62)$ & .29 \\
\hline
\end{tabular}

Values are given as number (percentage). BMI, Body mass index; $C H F$, congestive heart failure.

29.9. Patient demographics, tumor characteristics, and types of surgery performed were similar in 2 groups of BMI $(<25$ and $>=25)$, as shown in Table 1.

\section{Morbidities From Surgery}

The morbidities encountered perioperatively are shown in Table 2. A total of $62(19.3 \%)$ perioperative morbidities were encountered in 320 patients, with no significant difference in the rate of overall and specific morbidities $(P>.05$ in all cases) in between the 2 groups. The distributions of these morbidities between the 2 BMI groups are shown in Table 2 .

The median hospital stay in the normal BMI group was 5 days compared with 4 days $(P=.52$; Table 3$)$ in the higher BMI group. The median chest tube duration was the same 3 days across both the groups $(P=.052$; Table 3$)$.

\section{Mortality From Surgery}

The mortality rate in our population was $1.8 \%(n=6)$ (Table 4). The causes of death were different for all of them, and only 2 of the people who died had a BMI greater than 25 . The overall survival was similar between the 2 groups $(P=.46)$ (Figure 2).

\section{Interpretation of Multivariate Results (Table 5)}

Any perioperative morbidity. Forced expiratory volume in 1 second $\left(\mathrm{FEV}_{1}\right)$ was the only significant predictor of morbidity considered overall. For each 10-point decrease in $\mathrm{FEV}_{1}$, the odds of any sort of morbidity increased by approximately $14 \%(95 \% \mathrm{CI}, 2 \%-25 \% ; P=.0228)$. Although COPD was significant in univariate analysis $(P=.0253)$, COPD was no longer significant in multivariate analysis controlling for $\mathrm{FEV}_{1}(P=.2001)$.

Air leak. Among the factors considered, including a considerable effort to detect nonlinear effects of BMI, the

TABLE 3. Hospital and chest tube durations

\begin{tabular}{lccc}
\hline \multicolumn{1}{c}{ Variable } & BMI $<\mathbf{2 5}$ & BMI $\geq \mathbf{2 5}$ & $\boldsymbol{P}$ value \\
\hline Median hospital duration, d & 5 & 4 & .52 \\
Median chest tube duration, d & 3 & 3 & .052 \\
\hline
\end{tabular}

BMI, Body mass index.
TABLE 4. The 30-day mortality (causes)

\begin{tabular}{lcl}
\hline \multicolumn{1}{c}{ Causes } & BMI & Surgery types \\
\hline Pulmonary embolism/CHF & 27.5 & Lobectomy \\
Hypotension/ischemic bowel & 24.8 & Lobectomy \\
Renal failure & 24.8 & Lobectomy \\
GI bleed & 23.1 & Lobectomy \\
Suicide & 19.7 & Lobectomy \\
Cerebrovascular accident & 25.3 & Wedge resection \\
\hline BMI, Body mass index; $C H F$, congestive heart failure; $G I$, gastrointestinal.
\end{tabular}

only significant predictor was BMI lower than 20 versus BMI of 20 or higher, with the BMI of 20 or higher group having considerable lower odds of an air-leak morbidity (OR, 0.26; 95\% CI, 0.12-0.58; $P=.0011$ ). This effect was not significant when considering BMI lower than 25 versus $\mathrm{BMI}$ of 25 or higher $(P=.5035)$. We consider the BMI lower than 20 versus 20 or higher result to be a post hoc test. It is reported for completeness, but it does not relate directly to our main result about high BMI and morbidity.

Atrial fibrillation. No significant predictors were found.

Respiratory failure. COPD was the only significant predictor of respiratory failure morbidity on univariate and multivariate analysis $(\mathrm{OR}, 4.1 ; 95 \% \mathrm{CI}, 1.9-9.0 ; P=.0008)$.

\section{DISCUSSION}

To further describe the surgical risks of overweight patients undergoing resections for lung cancer, we performed a retrospective, single-institution review. Our series identified no difference between the morbidities encountered postsurgically and in mortality between overweight and normal weight patients. However, there could be small associations, which are present, but too weak to be detected. BMI did not have any effect on the overall and specific perioperative morbidities, whether as a linear (continuous)

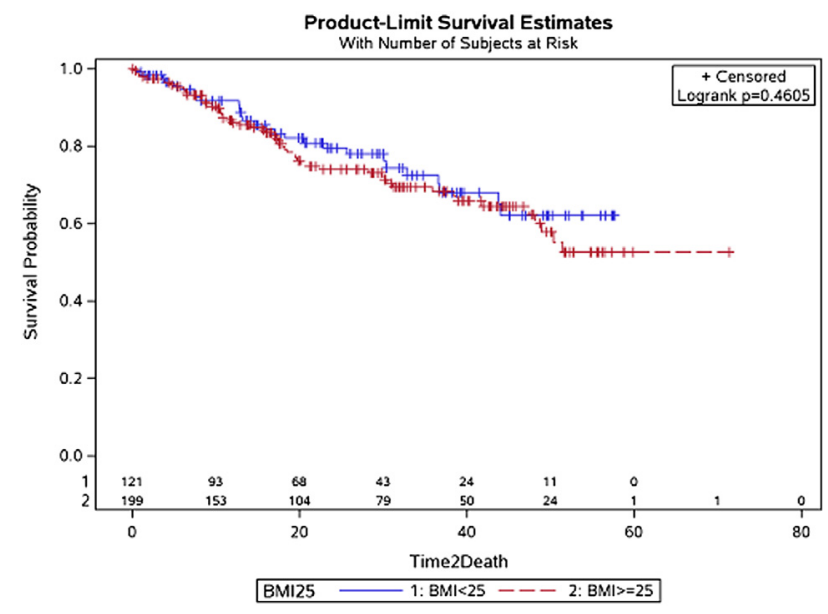

FIGURE 2. Kaplan-Meier survival curve showing no significant difference in the survival between the 2 groups. BMI, Body mass index. 
TABLE 5. Univariate and multivariate analysis of all and specific perioperative morbidities with different predictors

\begin{tabular}{|c|c|c|c|c|}
\hline Outcome & Predictors & OR $(95 \%$ CI $)$ & Univariate $P$ values & Multivariate $P$ values \\
\hline \multirow[t]{12}{*}{ Any morbidities } & $\operatorname{COPD}(\mathrm{Y}$ vs $\mathrm{N})$ & $1.85(1.09-3.15)$ & .0253 & .2001 \\
\hline & $\mathrm{CHF}(\mathrm{Y}$ vs N) & $0.72(0.34-1.55)$ & .4614 & .1544 \\
\hline & Sex (M vs F) & $1.02(0.63-1.63)$ & 1.0000 & .9376 \\
\hline & Surgery procedure & & .7887 & $.6734(d f=2)$ \\
\hline & BMI (linear) & $0.98(0.95-1.02)$ & .3095 & .3944 \\
\hline & $\mathrm{BMI}(d f=5)$ & & .5053 & $.5687(d f=5)$ \\
\hline & BMI25 $(25+$ vs $<25)$ & $0.84(0.52-1.35)$ & .5405 & .5879 \\
\hline & BMI20 $(20+$ vs $<20)$ & $0.49(0.24-1.00)$ & .0513 & .0683 \\
\hline & $\mathrm{FEV}_{1}$ (per 1-U FEV) & $0.98(0.97-1.00)$ & .0228 & .0228 \\
\hline & $\mathrm{FEV}_{1}($ per 10-U FEV) & $0.86(0.75-0.98)$ & .0228 & .0228 \\
\hline & $\mathrm{DLCO}($ per $1 \mathrm{U})$ & $0.99(0.98-1.01)$ & .3144 & .9945 \\
\hline & Age (per y) & $1.01(0.99-1.03)$ & .5367 & .7376 \\
\hline \multirow[t]{11}{*}{ Air leak } & $\operatorname{COPD}(\mathrm{Y}$ vs N$)$ & $1.45(0.71-2.93)$ & .3382 & .3969 \\
\hline & $\mathrm{CHF}(\mathrm{Y}$ vs N) & $0.52(0.15-1.77)$ & .4450 & .2800 \\
\hline & $\operatorname{Sex}(M$ vs F) & $1.05(0.55-1.99)$ & 1.0000 & .9461 \\
\hline & Surgery procedure & & .0998 & $.3295(d f=2)$ \\
\hline & BMI (linear) & $0.97(0.92-1.02)$ & .2731 & .2731 \\
\hline & $\mathrm{BMI}(d f=5)$ & & .0400 & $*$ \\
\hline & BMI25 $(25+$ vs $<25)$ & $0.77(0.40-1.47)$ & .5035 & Already in MV model \\
\hline & BMI20 $(20+$ vs $<20)$ & $0.26(0.12-0.58)$ & .0011 & $*$ \\
\hline & $\mathrm{FEV}_{1}($ per 1-U FEV) & $0.99(0.98-1.01)$ & .4876 & .6211 \\
\hline & DLCO (per $1 \mathrm{U})$ & $1.00(0.98-1.01)$ & .5914 & .7174 \\
\hline & Age (per y) & $1.00(0.97-1.03)$ & .9867 & .9402 \\
\hline \multirow[t]{11}{*}{ Atrial fibrillation } & COPD (Y vs N) & $1.15(0.51-2.57)$ & .8325 & \\
\hline & $\mathrm{CHF}(\mathrm{Y}$ vs N$)$ & $0.99(0.33-2.97)$ & 1.0000 & \\
\hline & $\operatorname{Sex}(M$ vs F) & $0.98(0.65-1.47)$ & 1.0000 & \\
\hline & Surgery procedure & & $.0990(d f=2)$ & \\
\hline & BMI (linear) & $1.01(0.96-1.06)$ & .6344 & \\
\hline & $\mathrm{BMI}(d f=5)$ & & .6320 & \\
\hline & BMI25 (25+ vs <25) & $1.37(0.65-2.91)$ & .4639 & \\
\hline & BMI20 (20+ vs < 20) & $0.88(0.29-2.66)$ & .8181 & \\
\hline & $\mathrm{FEV}_{1}$ (per 1-Unit FEV) & $0.99(0.97-1.01)$ & .2530 & \\
\hline & DLCO (per $1 \mathrm{U})$ & $1.00(0.98-1.02)$ & .7790 & \\
\hline & Age (per y) & $1.02(0.98-1.05)$ & .3862 & \\
\hline \multirow[t]{10}{*}{ Respiratory failure } & COPD (Y vs N) & $4.13(1.89-9.01)$ & .0008 & .0008 \\
\hline & $\mathrm{CHF}(\mathrm{Y}$ vs N) & $1.25(0.41-3.82)$ & .7589 & .7104 \\
\hline & Sex (M vs F) & $1.31(0.61-2.82)$ & .5558 & .5659 \\
\hline & Surgery procedure & & .7809 & .7068 \\
\hline & BMI (linear) & $0.98(0.93-1.05)$ & .6122 & .8222 \\
\hline & $\mathrm{BMI}(d f=5)$ & & .8557 & .8630 \\
\hline & BMI25 $(25+$ vs $<25)$ & $0.85(0.39-1.84)$ & .6917 & .8794 \\
\hline & $\mathrm{FEV}_{1}$ (per 1-U FEV) & $0.98(0.96-1.00)$ & .1095 & .7572 \\
\hline & DLCO (per $1 \mathrm{U})$ & $1.00(0.98-1.03)$ & .7444 & .1751 \\
\hline & Age (per y) & $1.01(0.97-1.05)$ & .6074 & .8353 \\
\hline
\end{tabular}

Multivariate analysis not performed for atrial fibrillation because there are no significant predictors. $O R$, Odds ratio; $C I$, confidence interval; $C O P D$, chronic obstructive pulmonary disease; $Y$, yes; $N$, no; $C H F$, congestive heart failure; $M$, male; $F$, female; $D L C O$, diffusing capacity for carbon monoxide; $M V$, multivariate; $B M I$, body mass index; $F E V$, forced expiratory volume. *Only in place of BMI 25.

variable or a dichotomous variable with 25 as the cutoff (Table 5). Our analysis also divided BMI into 4 different groups as per World Health Organization classification, with no significant difference in the overall morbidities in these groups except for the air leak, which tends to occur more in the lower BMI groups (Table 5).

In the logistic regression model considering any perioperative morbidity, after controlling for $\mathrm{FEV}_{1}$
$(P=.0228)$, continuous BMI is not significant $(P=.3944)$. Receiver operating characteristic (curve) plots show no difference in the model, including both $\mathrm{FEV}_{1}$ and $\mathrm{BMI}$, compared with $\mathrm{FEV}_{1}$ alone. There is no indication that BMI at any cut point would add significant predictive power.

In the logistic regression model considering air leak, continuous BMI is not significant $(P=.2731)$. The receiver 
operating characteristic (curve) plot for BMI maximizes the sum of sensitivity and specificity at approximately 24 to 25 BMI, but there is no significant predictive power (Figure 3).

The duration of hospital stay is determined by many factors, such as wound healing, comorbidities, and physical deconditioning from surgery. Our study suggests that a higher BMI does not have any effect on the duration of hospital stay, because the median length of hospital stay was the same between the 2 groups. The median chest tube duration was 3 days in both the groups; however, because of the higher incidence of outliers in the nonobese groups, the $P$ value approached the level of significance. This should be interpreted with caution because this number is highly influenced by a few patients with a high value of chest tube days, and is not adjusted for multiple comparisons. Length of stay and even chest tube duration are influenced by many factors, including surgeon preference, variable discharge criteria, and varying protocols for chest tube management, along with other social factors.

The outcome of our study is similar to the study by Smith et al, ${ }^{18}$ in which no difference in overall morbidity, mortality, and length of stay was found. The mortality rate in their study was $1.4 \%$, slightly lower than our $1.8 \%$ mortality rate, but both were within the accepted standards. In the study by Smith et al, the patients were divided into 2 groups of BMI $(<30$ and $\geq 30)$. Our study is different from that because we divided our patients into 2 groups, with a cutoff BMI of 25 , because we decided to study the risks of overweight

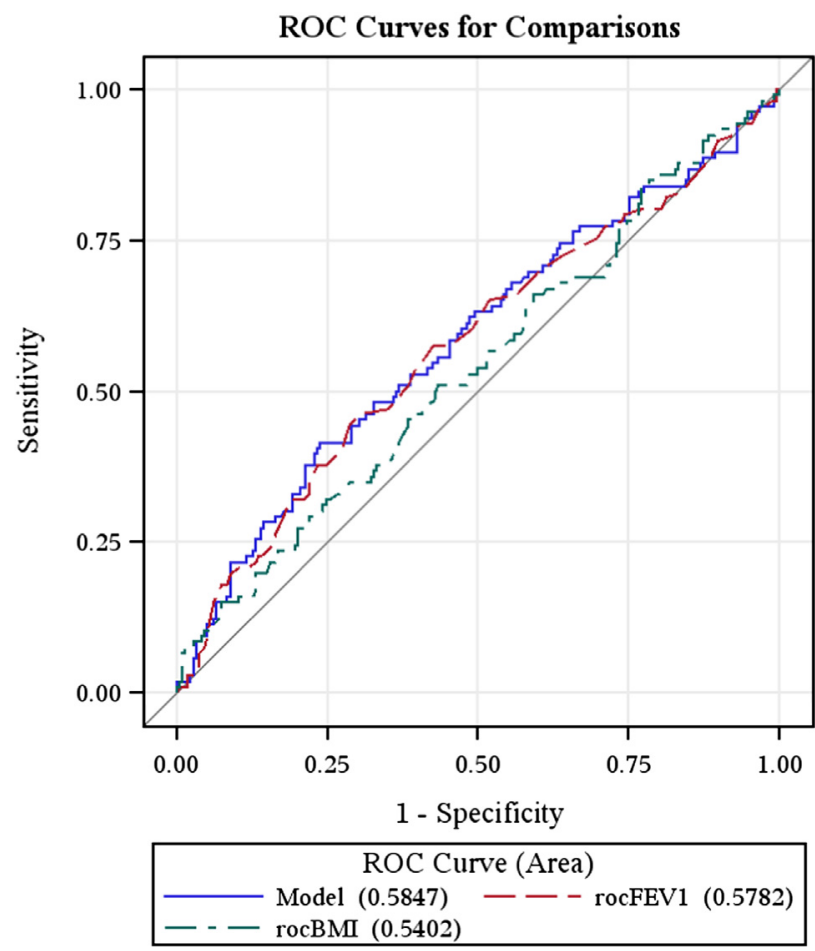

FIGURE 3. Receiver operating characteristic (ROC) curve for model, body mass index $(B M I)$, and forced expiratory volume in 1 second (FEVI). population versus those who have a normal BMI. Analysis of our data using the cutoffs used by Smith et al did not change our results. In the same study, it was shown that obese patients were at higher risk of developing renal failure $(0.3 \%$ vs $3.9 \% ; P=.001)$. However, this was not confirmed in our series. They also showed a decreased tendency for respiratory complications in obese patients $(21.8 \%$ vs $14.2 \%$; $P=.06$ on bivariate analysis) because of higher diffusing capacity for carbon monoxide and low smoking rates in obese patients. Our study showed the opposite trend, because there was no significant difference in respiratory complications when compared with patients with a normal BMI $(9.94 \%$ vs $8.54 \% ; P=.69)$. $\mathrm{FEV}_{1}$ was the only significant predictor of morbidity considered overall.

In contrast, another study by Petrella et $\mathrm{al}^{20}$ showed that overweight and obese patients who underwent pneumonectomies for lung cancer had a higher risk of respiratory complications compared with patients with a normal BMI $(21.4 \%$ vs $4.9 \% ; P=.005)$. The study, however, did not show any difference in cardiac complications, 30-day mortality, and length of stay between the 2 groups. ${ }^{20}$ Our study was not limited to pneumonectomies; only 14 patients with such a procedure were included as part of our cohort. No differences in outcomes between normal and overweight patients were seen in this subgroup in our series.

Our study is limited by its retrospective nature; it is possible that selection bias is responsible for our findings. The sample size is also small in our study to detect the meaningful differences between the 2 groups. We also did not take into account the other factors that can affect the morbidity and mortality, such as diabetes, hypertension, and underlying lung disease, among others, and it was limited to COPD and congestive heart failure only. Moreover, in our series, the sample size is even smaller when we use a higher BMI ( $>35)$ to see if the differences exist. We also did not examine the baseline nutritional status of our patients, such as prealbumin/albumin, which might influence the overall outcome. Our study did not examine the outcomes with minimal invasive surgery and the extent of resection. In our multivariate analysis, we took into account the $3 \mathrm{ma}-$ jor types of surgery and found no difference in outcomes with the types of surgery. Over a 5-year period, the changing approach for lung resection would have influenced the perioperative outcomes and our study was limited to that aspect. Large multi-institutional and prospective studies are needed to fully elucidate the effects of higher BMI because of several limitations of our retrospective study.

Because of the changing lifestyles, both BMI and lung cancer are increasing. In the future, thoracic surgeons are likely to encounter more patients with a higher BMI with non-small cell lung cancer. Our study suggests that a higher BMI does not carry significant risk for lung cancer surgery. However, large, multi-institutional, and prospective studies are needed to support this evidence. 


\section{References}

1. Flegal KM, Carroll MD, Ogden CL, Johnson CL. Prevalence and trends in obesity among US adults, 1999-2000. JAMA. 2002;288:1723-7.

2. DeMaria EJ, Carmody BJ. Perioperative management of special populations: obesity. Surg Clin North Am. 2005;85:1283-9. xii.

3. Raebel MA, Malone DC, Conner DA, Xu S, Porter JA, Lanty FA. Health services use and health care costs of obese and nonobese individuals. Arch Intern Med. 2004;164:2135-40.

4. Larsson SC, Orsini N, Wolk A. Body mass index and pancreatic cancer risk: a meta-analysis of prospective studies. Int J Cancer. 2007;120:1993-8.

5. Renehan AG, Tyson M, Egger M, Heller RF, Zwahlen M. Body-mass index and incidence of cancer: a systematic review and meta-analysis of prospective observational studies. Lancet. 2008;371:569-78.

6. Pi-Sunyer FX. Medical hazards of obesity. Ann Intern Med. 1993;119:655-60.

7. Ramsey AM, Martin RC. Body mass index and outcomes from pancreatic resection: a review and meta-analysis. J Gastrointest Surg. 2011;15:1633-42.

8. Wigfield CH, Lindsey JD, Munoz A, Chopra PS, Edwards NM, Love RB. Is extreme obesity a risk factor for cardiac surgery? an analysis of patients with a BMI $>$ or $=40$. Eur J Cardiothorac Surg. 2006;29:434-40.

9. Klasen J, Junger A, Hartmann B, Jost A, Benson M, Virabjan T, et al. Increased body mass index and peri-operative risk in patients undergoing non-cardiac surgery. Obes Surg. 2004;14:275-81.

10. Mullen JT, Davenport DL, Hutter MM, Hosokawa PW, Henderson WG, Khuri SF, et al. Impact of body mass index on perioperative outcomes in patients undergoing major intra-abdominal cancer surgery. Ann Surg Oncol. 2008;15:2164-72.

11. Thomas EJ, Goldman L, Mangione CM, Marcantonio ER, Cook EF, Ludwig L, et al. Body mass index as a correlate of postoperative complications and resource utilization. Am J Med. 1997;102:277-83.
12. Romano PS, Mark DH. Patient and hospital characteristics related to in-hospital mortality after lung cancer resection. Chest. 1992;101:1332-7.

13. Stolz AJ, Schutzner J, Lischke R, Simonek J, Pafko P. Predictors of prolonged air leak following pulmonary lobectomy. Eur J Cardiothorac Surg. 2005;27: 334-6.

14. Allen MS, Darling GE, Pechet TT, Mitchell JD, Herndon JE II, Landreneau RJ, et al. Morbidity and mortality of major pulmonary resections in patients with early-stage lung cancer: initial results of the randomized, prospective ACOSOG Z0030 trial. Ann Thorac Surg. 2006;81:1013-9; discussion 1019-20.

15. Berggren H, Ekroth R, Malmberg R, Naucler J, William-Olsson G. Hospital mortality and long-term survival in relation to preoperative function in elderly patients with bronchogenic carcinoma. Ann Thorac Surg. 1984;38:633-6.

16. Damhuis RA, Schutte PR. Resection rates and postoperative mortality in 7,899 patients with lung cancer. Eur Respir J. 1996;9:7-10.

17. Weiss W. Operative mortality and five year survival rates in patients with bronchogenic carcinoma. Am J Surg. 1974;128:799-804.

18. Smith PW, Wang H, Gazoni LM, Shen KR, Daniel TM, Jones DR. Obesity does not increase complications after anatomic resection for non-small cell lung cancer. Ann Thorac Surg. 2007;84:1098-105; discussion 1105-6.

19. Suemitsu R, Sakoguchi T, Morikawa K, Yamaguchi M, Tanaka H, Takeo S. Effect of body mass index on perioperative complications in thoracic surgery. Asian Cardiovasc Thorac Ann. 2008;16:463-7.

20. Petrella F, Radice D, Borri A, Galetta D, Gasparri R, Solli P, et al. The impact of preoperative body mass index on respiratory complications after pneumonectomy for non-small-cell lung cancer: results from a series of 154 consecutive standard pneumonectomies. Eur J Cardiothorac Surg. 2011;39:738-44. 